\title{
Risk factors for developing clinical infection with methicillin-resistant Staphylococcus aureus (MRSA) amongst hospital patients initially only colonized with MRSA
}

\author{
R. Coello*, J. R. Glynn†, C. Gaspar*, J. J. Picazoł and J. Fereres* \\ *Servicio de Medicina Preventiva and $\$$ Servicio de Microbiologia Clínica, \\ Hospital Universitario San Carlos, Madrid, Spain; and †Communicable \\ Disease Epidemiology Unit, Department of Epidemiology and Population \\ Sciences, London School of Hygiene and Tropical Medicine, London WC1E \\ $7 H T, U K$
}

Received 20 February 1997; revised manuscript accepted 28 May 1997

\begin{abstract}
Summary: In hospital outbreaks of methicillin-resistant Staphylococcus aureus (MRSA) many patients are initially colonized without infection. The reasons why some progress to infection while others do not are not known. A cohort of 479 hospital patients, initially only colonized with MRSA, was followed prospectively for the development of MRSA infection. Risk factors for progression to infection were assessed using Cox proportional hazards survival analysis. Fifty-three patients $(11 \cdot 1 \%)$ developed 68 MRSA infections. Intensive care setting, administration of three or more antibiotics, ulcers, surgical wounds, nasogastric or endotracheal tubes, drains, and urinary or intravenous catheterization were all associated with increased rates of MRSA infection. Multivariate analysis showed that intensive care patients, compared with medical patients, had a higher rate of developing MRSA infection within the first four days of admission, with a hazard ratio of 26.9 (95\% CI 5.7-126). Surgical wounds, pressure ulcers and intravenous catheterization were also independent risk factors, with hazard ratios (and $95 \% \mathrm{CI}$ ) of 2.9 $(1 \cdot 3-6 \cdot 3), 3 \cdot 0(1 \cdot 6-5 \cdot 7)$ and $4 \cdot 7(1 \cdot 4-15 \cdot 6)$, respectively. These findings suggest that, during an MRSA outbreak, clinical infection would be reduced if surgical and intensive care patients received priority for the prevention of initial colonization with MRSA. Prevention of pressure ulcers, and strict aseptic care of intravenous catheters and surgical wounds would also reduce the development of MRSA infection. Since early treatment with vancomycin is known to reduce the mortality, patients colonized with MRSA who also have one or more of these risk factors may warrant empirical vancomycin therapy at the carliest suggestion of infection.
\end{abstract}

Keywords: MRSA; risk factors; hospital infection; colonization.

\section{Introduction}

It has been known for many years that the early detection and treatment of asymptomatic carriers contributes to the control of epidemic Staphylocaccus aureus, including methicillin-resistant strains (MRSA). ${ }^{1,2}$ Broken skin, the

Correspondence to: $\mathrm{Dr}$ Rosa Coello. Present affiliation: Nosocomial Infection Surveillance Unit, Central Public Health Laboratory, 61 Colindale Avenue, London NW9 5HT, UK. 
respiratory tract and the urinary tract may become asymptomatically colonized with MRSA. In addition, MRSA may colonize normal carrier sites such as the nose, throat, perineum, groin and axillae. Colonized patients may subsequently developed clinical infection, ${ }^{3}$ but there are no published studies to indicate which colonized patients with MRSA are most vulnerable to infection. Knowledge of the factors associated with the development of MRSA infection would indicate which patients to target for the prevention of acquisition of MRSA. Amongst those patients already colonized, it may be possible to control some risk factors and thus prevent subsequent MRSA infection.

From November 1989 to October 1992, a large outbreak of MRSA affected 990 patients at Iospital Universitario San Carlos in Madrid. The details have already been published elsewhere. ${ }^{3}$ We identified a cohort of patients who were initially only colonized with MRSA and determined the factors associated with the subsequent development of clinical infection.

\section{Methods}

Hospital Universitario San Carlos is a 1500-bed teaching hospital covering all major specialities and serving a population of 600000 . The study population consisted of all patients identified as being colonized but not infected with hospital-acquired MRSA in the two years from November 1990. They were followed up prospectively until discharge from hospital.

Methicillin-resistant Staphylococcus aureus were isolated and identified using standard microbiological methods. ${ }^{4}$

Hospital-acquired MRSA was defined as the isolation of MRSA $48 \mathrm{~h}$ or more after admission from patients without previous hospitalization or previous MRSA during the preceding year. Infected patients were defined according to the Centers for Disease Control standard definitions for specific infections. ${ }^{5}$ Colonized patients were defined as those from whom MRSA was isolated from any body site but without clinical symptoms of infection, including those patients from whom MRSA was isolated from normal carrier sites (the anterior nares, throat, perineum, groin or axilla).

Routine clinical microbiological specimens were used to identify infected and colonized patients. In addition, screening specimens were taken from carriage sites of room-mates of known MRSA patients, and from patients who were exposed to staff known to have MRSA according to the UK guidelines for the control of epidemic MRSA. ${ }^{1}$ The clinical records of each patient were reviewed to distinguish MRSA infection from colonization.

Possible risk factors for the development of MRSA infection were identified by visiting the patients and by chart review. The following factors were considered: age, sex, number of underlying diagnoses, insulin dependent diabetes, hospital department at the time of MRSA isolation, the interval between the acquisition of MRSA colonization and the development of MRSA clinical infection, other infections or antibiotic 
therapy in the month preceding MRSA isolation, the presence of pressure ulcers, vascular ulcers, surgical wounds, other skin lesions, or tracheostomy at the time of MRSA isolation or in the $72 \mathrm{~h}$ preceding MRSA isolation, and invasive devices such as drains, nasogastric or endotracheal tubes, urinary-tract or intravascular catheters that had been in situ for more than $48 \mathrm{~h}$ within the $72 \mathrm{~h}$ preceding MRSA isolation.

The effects of the risk factors were assessed individually and then in a multivariate model, using Cox proportional hazards survival analysis. The proportional hazards assumption for each of the risk factors was tested by inspecting Kaplan-Meier plots and formal testing. If the hazards were found to be non-proportional the analysis was repeated using separate time periods defined by the median event time. Finally, interaction amongst the risk factors was assessed.

\section{Results}

For the two years from November 1990,776 patients were identified with hospital-acquired MRSA, of whom 479 were initially only colonized and constituted the cohort for this study. Of the 479 colonized patients, 53 $(11 \cdot 1 \%)$ subsequently developed 68 MRSA infections. Twelve patients $(22.6 \%)$ had more than one infection. The distribution of the infections was $22(32 \cdot 4 \%)$ surgical wound, nine $(13.2 \%)$ lower respiratory tract, nine $(13 \cdot 2 \%)$ urinary tract, eight $(11 \cdot 8 \%)$ pressure ulcers, six $(8 \cdot 8 \%)$ bacteraemias, four $(5.9 \%)$ vascular ulcers and $10(14.7 \%)$ other infections.

Table I shows the potential risk factors and the unadjusted hazard ratios, for developing MRSA infection in this cohort of patients. MRSA infection was associated with previous use of antibiotics, the presence of ulcers or surgical wounds, and the use of tubes, drains and catheters. For hospital department, the hazards were not proportional over time (Figure), the rate of infection being much greater early in the intensive care unit setting. Half of the infections occurred within 12 days, but the rate was still not proportional within this period. The median event time within the first 12 days was four days, and therefore was used to divide the time further.

In the multivariate analysis, hospital department was considered in two time periods ( $\leq$ four days and $>$ four days of admission). The hazard ratios for hospital department were hardly changed after controlling for the other potential risk factors shown in Table $\mathrm{I}$. Within the first four days of admission, compared with medical patients, those in the intensive care unit had an increased rate of MRSA infection with a hazard ratio of 26.9 , and $95 \%$ confidence intervals (CI) 5.7-126. However, after four days of admission, the hazards were proportional and stay in intensive care unit was no longer a risk factor for the development of MRSA infection (hazard ratio $1 \cdot 1$, and $95 \% \mathrm{CI} 0 \cdot 3-3 \cdot 9$ ). Admission to the surgical wards was not associated with an increased rate of MRSA infection, with a hazard ratio (and 95\% CI) within the first for days of 1.7 (0.3-10.1), and 1.7 (0.9-3.4) after the first four days. The effect of the other risk factors was also assessed 
Table I. Crude hazard ratios and 95\% confidence intervals (CIs) for developing MRSA infection according to several risk factors, in a cohort of 479 colonized patients with MRSA using Co. proportional hazards model

\begin{tabular}{|c|c|c|c|c|}
\hline \multirow[t]{2}{*}{ Risk factors } & \multicolumn{4}{|c|}{ No of patients: } \\
\hline & $\begin{array}{l}\text { Developed } \\
\text { infection } \\
(N=53)\end{array}$ & $\begin{array}{l}\text { Remained } \\
\text { colonized } \\
(N=426)\end{array}$ & $\begin{array}{l}\text { Hazard ratio } \\
\text { (and } 95 \% \mathrm{CI} \text { ) }\end{array}$ & $P$ \\
\hline Female & 20 & 153 & $0.98(0.56-1 \cdot 71)$ & 0.9 \\
\hline \multicolumn{5}{|l|}{ Age (years) } \\
\hline $16-24$ & 2 & 8 & 1 & \\
\hline $25-44$ & 5 & 20 & $1 \cdot 29(0 \cdot 25-6 \cdot 69)$ & $0 \cdot 8$ \\
\hline $45-64$ & 9 & 89 & $0.55(0 \cdot 12-2 \cdot 56)$ & 0.4 \\
\hline$\geq 65$ & 37 & 309 & $0.56(0.13-2 \cdot 34)$ & 0.4 \\
\hline Three or more diagnoses & 46 & 336 & $1.07(0.45-2 \cdot 50)$ & 0.9 \\
\hline Insulin dependant diabetes & 9 & 40 & $1.41(0.69-2.90)$ & $0 \cdot 3$ \\
\hline Other infections & 34 & 221 & $1 \cdot 48(0 \cdot 83-2 \cdot 65)$ & $0 \cdot 2$ \\
\hline \multicolumn{5}{|l|}{ Department } \\
\hline Medicine & 16 & 215 & 1 & \\
\hline Surgery & 26 & 185 & $1.76(0.94-3.29)$ & 0.08 \\
\hline Intensive care unit & 11 & 25 & $3.91(1.81-8.46)$ & $<0.001^{*}$ \\
\hline \multicolumn{5}{|l|}{ No of antibiotics } \\
\hline None & 3 & 70 & 1 & \\
\hline $1-2$ & 22 & 211 & $3 \cdot 16(0 \cdot 95-10 \cdot 6)$ & 0.06 \\
\hline$\geq 3$ & 25 & 128 & $4 \cdot 88(1 \cdot 47-16 \cdot 2)$ & 0.01 \\
\hline Skin lesions & 6 & 30 & $1.72(0.73-4.04)$ & $0 \cdot 2$ \\
\hline Tracheostomy & 1 & 23 & $0.23(0 \cdot 31-1 \cdot 65)$ & $0 \cdot 1$ \\
\hline Vascular ulcers & 9 & 23 & $2.04(0.99-4.30)$ & $0 \cdot 05$ \\
\hline Pressure ulcers & 20 & 59 & $2 \cdot 14(1 \cdot 21-3 \cdot 80)$ & 0.009 \\
\hline Surgical wounds & 29 & 132 & $2 \cdot 97(1 \cdot 72-5 \cdot 12)$ & $<0.001$ \\
\hline \multicolumn{5}{|l|}{ Invasive devices } \\
\hline Nasogastric tubes & 24 & 99 & $2 \cdot 25(1 \cdot 29-3 \cdot 93)$ & 0.004 \\
\hline Drains & 18 & 91 & $2 \cdot 30(1 \cdot 29-4 \cdot 13)$ & $0 \cdot 005$ \\
\hline Endotracheal tubes & 8 & 30 & $2 \cdot 37(1 \cdot 11-5 \cdot 10)$ & 0.03 \\
\hline Urinary tract catheters & 35 & 194 & $2 \cdot 49(1.34-4.62)$ & $0 \cdot 004$ \\
\hline Intravenous catheters & 48 & 250 & $8 \cdot 69(2 \cdot 70-27 \cdot 9)$ & $0 \cdot 001$ \\
\hline
\end{tabular}

* Non-proportional hazards. See text.

in the multivariate analysis and stratified for hospital department. Surgical wounds, pressure ulcers and intravenous catheterization were also independently associated with an increased rate of MRSA infection (Table II). The Kaplan-Meier plots showed that the proportional hazards assumption was satisfied for surgical wounds, pressure ulcers and intravenous catheterization (Figure). No interaction was found amongst the risk factors.

\section{Discussion}

Many of the risk factors associated with the development of MRSA infection are correlated. For example, intensive care is associated with invasive procedures, antibiotics with intravenous catheters, pressure ulcers with age. By studying a cohort of patients colonized with MRSA and by using 

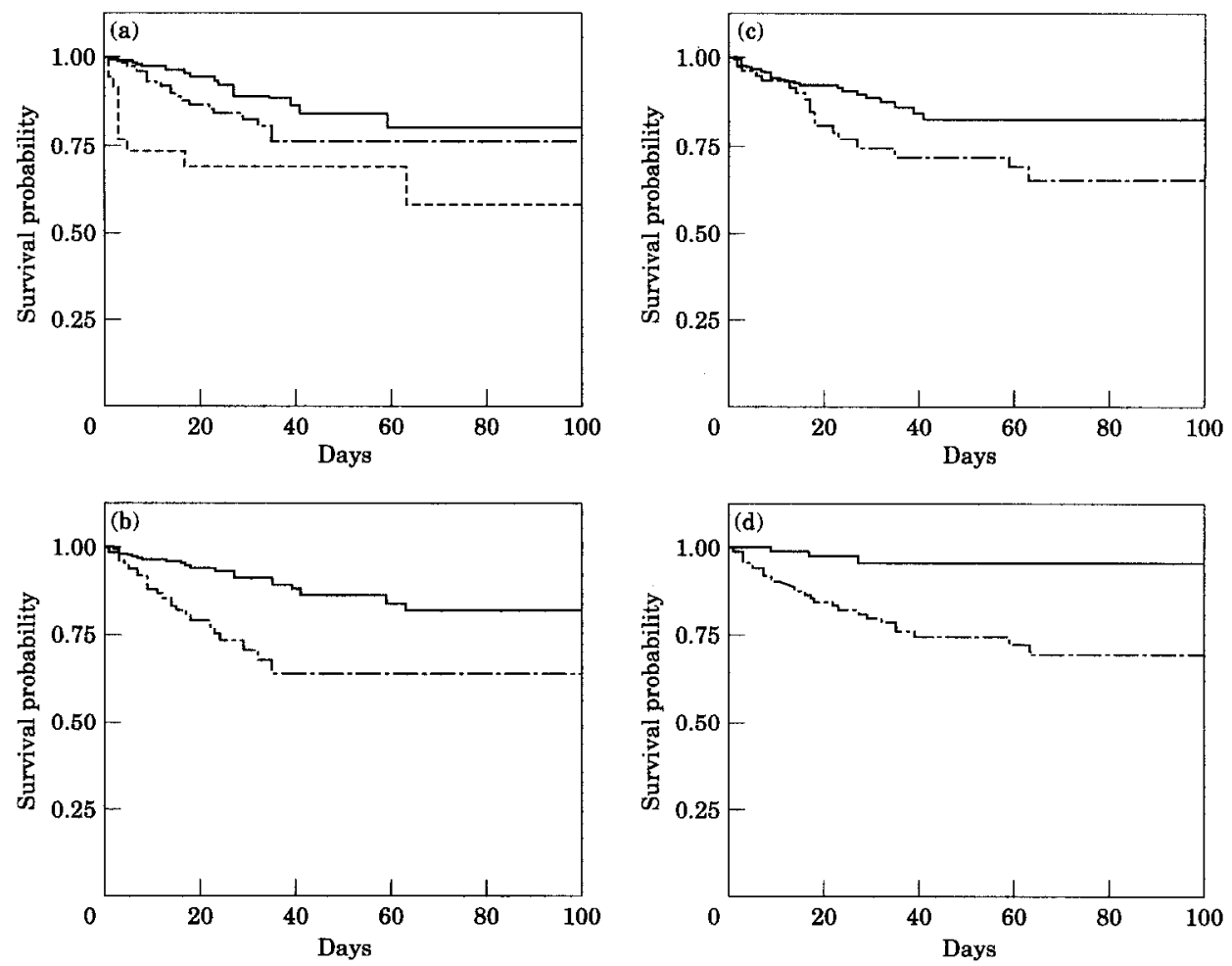

Figure 1. Kaplan-Meier curves for development of MRSA infection among colonized patients by: (a) hospital departments $\left[(-)\right.$ medicine; $(\cdots \cdots \cdot)$ intensive care; $\left(\cdot-\cdot{ }^{-}-\right)$ surgery]; (b) surgical wounds [(-) without; (-..--) with]; (c) pressure ulcers [(-) without; $\left(\cdot \cdot^{-\cdot \cdot-)}\right.$ with]; (d) intravenous catheters $\left[(-)\right.$ without; $\left(\cdot \cdot_{-} \cdot-\right)$ with]. The 'survival probability' ( $y$-axis) is the proportion of patients who are free of MRSA infection. The curves shown are censored at 100 days. By this time only 26 patients were still at risk and only three subsequently developed infection.

Table II. Independent risk factors for the development of MRSA infection in a cohort of 479 colonized patients, from a Cox proportional hazards model stratified by hospital department

\begin{tabular}{lcc}
\hline Risk factors & Adjusted* hazard ratios (and 95\% CIs) $^{*}$ & $P$ \\
\hline Surgical wound & $2.90(1 \cdot 31-6 \cdot 32)$ & 0.008 \\
Pressure ulcers & $3.03(1 \cdot 60-5 \cdot 73)$ & $<0.001$ \\
Intravenous catheter & $4.70(1 \cdot 41-15 \cdot 6)$ & 0.01 \\
\hline
\end{tabular}

* Adjusted for each of the risk factors shown in this table.

multivariate models, we have identified those risk factors which independently influence the subsequent development of MRSA infection. The use of Cox regression analysis took into account the time that individual patients were at risk and thus avoided potential bias due to colonized patients without clinical problems being discharged after a short stay in hospital. 
Eleven percent of our cohort of colonized patients subsequently developed MRSA infection, a proportion very similar to that recently observed by Bendall et $a l .{ }^{6}$ Our results suggest that, in addition to contributing to the control of spread of MRSA, identification of colonized patients is important as some are at special risk of developing infection. Of the 17 potential risk factors considered for the development of MRSA infection in colonized patients, only intensive care unit setting, surgical wounds, pressure ulcers and intravenous catheters were independently associated with an increased rate of subsequent MRSA infection.

Although intensive care patients acquire MRSA infection more often than other hospital patients, ${ }^{7-11}$ it has not been shown before that intensive care is a risk factor after controlling for other factors such as invasive devices. Our analysis showed that patients in intensive care had high rates of developing MRSA infection early in their stay. The increased rate among intensive care patients might be explained by the frequent opportunities for MRSA invasion arising from nursing contact, when MRSA can be transferred, via staff hands, from colonized sites to a portal of entry such as broken skin. This high rate early after colonization may reflect the presence of multiple risk factors in intensive care patients, such as intravenous catheters and wounds. Due to the severity of the illness in these patients, they are likely to be both more vulnerable to infection and to have more microbiological tests which would increase the chance of detecting MRSA infection earlier.

A high proportion of the patients in our cohort (28 of 53) developed infection in surgical wounds or pressure ulcers. The damaged skin in these colonized patients probably provides a portal of entry for MRSA to the underlying tissues which readily leads to local or generalized infection. It is known that patients with skin wounds are more often colonized with MRSA at other body sites, including wounds, ${ }^{12}$ and that $S$. aureus infecting surgical wounds is often derived from the patient's nose. ${ }^{13}$ Furthermore, once a wound is colonized with MRSA, the organisms tend to persist ${ }^{10,12}$ and are more likely to cause surgical wound infection than methicillinsensitive strains. ${ }^{7}$ Whilst it may not be possible to prevent all pressure ulcers, more appropriate nursing care for those patients who are known to be at risk of pressure ulcers ${ }^{14}$ would decrease MRSA infection. Although, surgical wounds cannot be prevented, it may be possible to prevent colonization by more thorough preoperative preparation of the patient's skin and wound care. The eradication of nasal MRSA with preoperative topical mupirocin may also contribute to the prevention of postoperative wound infection with MRSA. ${ }^{15}$

The presence of one or more intravenous catheters was a strong risk factor for the development of MRSA infection in colonized patients, with a hazard ratio of 4.7 after controlling for confounding. Pujol et al., ${ }^{16}$ in a study restricted to MRSA bacteraemia, also found that intravenous 
catheterization was an independent risk factor. Why intravenous catheterization should be a risk factor for the development of MRSA infection other than bacteraemia is not clear. Possibly patients with intravenous lines have more contacts with ward personnel which provide more opportunities for the transfer of MRSA, via staff hands, from colonized sites to other sites such as wounds or vulnerable damaged skin. The risk posed by intravenous catheters emphasizes the importance of aseptic catheter insertion, catheter care and early catheter removal, possibly with the assistance of specialized intravenous therapy teams. Appropriate care of intravenous catheters should decrease the risk of MRSA bacteraemia.

Although patients on intensive care units with intravenous catheters are likely to have more severe underlying illnesses, we do not believe that this is the major reason that they were associated with increased rates of MRSA infection, since no association was found with other indicators of patient vulnerability such as the number of diagnoses, or age.

The administration of more than three antibiotics was associated with the development of MRSA infection amongst patients already colonized with MRSA, but this effect did not persist after adjusting for the other risk factors. Previous studies have shown that when patients infected with MRSA were compared with those infected with methicillin-sensitive $S$. aureus, the number of antibiotics received and the duration of the antibiotic therapy were statistically associated with an increased risk of MRSA infection. ${ }^{7-9}$ Taken with our findings, this suggests that the number of antibiotics may be important in promoting the colonization of patients with MRSA but, once colonized with MRSA, the administration of multiple antibiotics does not pose an independent risk of subsequent MRSA infection.

Urinary tract catheters, nasogastric tubes and endotracheal tubes were not independent risk factors for MRSA infection and it could be argued that these factors differ from intravenous catheters in that there is usually no damage to the skin or mucus membrane which may be necessary to promote infection. The reason why other sites with broken skin such as tracheostomy, vascular ulcers and other skin lesions were not identified as risk factors for the development of MRSA infection might be explained by the relatively small numbers of patients with these factors.

At a time when controlling the epidemic spread of MRSA is becoming increasingly difficult and expensive, it is important to identify patients at particular risk. Our results suggest that surgical and intensive care patients should receive priority for infection control resources in order to prevent the introduction and spread of MRSA since, once colonized, they are more likely to develop infection. Early treatment with vancomycin in patients with MRSA infection is known to reduce the mortality. ${ }^{17}$ Thus, in order to reduce mortality associated with MRSA infection, patients colonized with MRSA who also have one or more of the risk factors that we have identified may warrant prompt empirical treatment with vancomycin if there is any suggestion of clinical infection. 
We thank Professor Mark Casewell of King's College School of Medicine and Dentistry for his help with the preparation of this manuscript. This study would have not been possible without the infection control team of Hospital Universitario San Carlos. This study was supported by a grant (FIS 94/5012) from Fondo de Investigación Sanitaria, Ministerio de Sanidad y Consumo, Madrid, Spain. Presented in part: 35 th Interscience Conference on Antimicrobial Agents and Chemotherapy, 17-20 September 1995, San Francisco, California (Abstract J134).

\section{References}

1. Report of a Combined Working Party of the Hospital Infection Society and British Society for Antimicrobial Chemotherapy. Revised guidelines for the control of epidemic methicillin-resistant Staphylococcus aureus. F Hosp Infect 1990; 6: 351-377.

2. Vandenbroucke-Grauls CM, Frenay HM, Van Klingeren B, Savelkoul TF, Verhoef J. Control of epidemic methicillin-resistant Staphylococcus aureus in a Dutch university hospital. Eur f Clin Microbiol Infect Dis 1991; 10: 6-11.

3. Coello R, Jiménez J, García M, Arroyo P, Mínguez D, Fernández C, Cruzet F, Gaspar C. Prospective study of infection, colonisation and carriage of methicillin-resistant Staphylococcus aureus in an outbreak affecting 990 patients. Eur $\mathcal{F}$ Clin Microbiol Infect Dis 1994; 13: 74-81.

4. National Committee for Clinical Laboratory Standards. Performance standards for antimicrobial disk susceptibility test. Tentative standards M2-T3. National Committee for Clinical Laboratory Standards, Villanova, PA, USA, 1987.

5. Garner JS, Jarvis WR, Emori TG, Horan TC, Hughes JM. CDC definitions for nosocomial infection, 1988. Am F Infect Control 1988; 16: 128-140.

6. Bendall RP, González-Ruiz A, Batiste L, Kelsey MC. Outcome of methicillin-resistant Staphylococcus aureus carriage. Lancet 1994; 344: 819.

7. Crossley K, Loesch D, Landesman B, Mead K, Chern M, Strate R. An outbreak of infections caused by strains of Staphylococcus aureus resistant to methicillin and aminoglycosides. I. Clinical Studies. F Infect Dis. 1979; 139: 273-279.

8. Peacock JE Jr, Marsik FJ, Wenzel RP. Methicillin-resistant Staphylococcus aureus: introduction and spread within a hospital. Ann Intern Med 1980; 93: 526-532.

9. Boyce JM, Laundry M, Dectz TR, Dupont HL. Epidemiologic studies of an outbreak of nosocomial methicillin-resistant Staphylococuus aureus infections. Infect Control 1981; 2: $110-116$.

10. Thompson RL, Cabezudo I, Wenzel RP. Epidemiology of nosocomial infections caused by methicillin-resistant Staphylococcus aureus. Ann Intern Med 1982; 97: 309-317.

11. Valls V, Gómez-Herruz P, Gómez-Palacios R, Cuadros JA, Romanyk JP, Ena J. Longterm efficacy of a program to control methicillin-resistant Staphylococcus aureus. Eur $\mathcal{f}$ Clin Microbiol Infect Dis 1994; 13: 90-95.

12. Bradley SF, Terpenning MS, Ramsey MA, Zarins LT, Jorgense KA, Sottile WS, Schaberg DR, Kauffman CA. Methicillin-resistant Staphylococcus aureus: colonization and infection in a long-term care facility. Ann Intern Med 1991; 115: 417-22.

13. Williams REO, Jevons MP, Shooter RA, Hunter CJW, Girling JA, Griffiths JD, Taylor GW. Nasal staphylococci and sepsis in hospital patients. $B M \mathcal{M}$ 1958; 2 : 658-662.

14. Panel for the Prediction and Prevention of Pressure Ulcers in Adults. Pressure Ulcers in Adults: Prediction and prevention. Clinical practice Guideline Number 3. Agency for Health Care Policy and Research, Public Health Service, US Department of Health and Human Services. May 1992.

15. Wenzel RP, Perl TM. The significance of nasal carriage of Staphylococcus aureus and the incidence of postoperative wound infection. F Hosp Infect 1995; 31: 13-24.

16. Pujol M, Peña C, Pallares R, Ayats J, Ariza J, Gudiol F. Risk factors for nosocomial bacteraemia due to methicillin-resistant Staphylococcus aureus. Eur $\mathcal{F}$ Clin Microbiol Infect Dis 1994; 13: 96-102.

17. French GL, Cheng AF, Ling JM, Mo P, Donnan S. Hong Kong strains of methicillinresistant and methicillin-sensitive Straphylococcus aureus have similar virulence. $\mathcal{F}$ Hosp Infect 1990; 15: 117-125. 